\title{
Analysis of gallstone disease after gastric cancer surgery
}

\author{
Tsung-Jung Liang ${ }^{1} \cdot$ Shiuh-Inn Liu ${ }^{1,2} \cdot$ Yu-Chia Chen ${ }^{1} \cdot$ Po-Min Chang ${ }^{1} \cdot$ \\ Wei-Chun Huang ${ }^{2,3} \cdot$ Hong-Tai Chang ${ }^{1,2} \cdot$ I-Shu Chen ${ }^{1}$ (i)
}

Received: 17 July 2016/ Accepted: 23 January 2017

(c) The International Gastric Cancer Association and The Japanese Gastric Cancer Association 2017

\begin{abstract}
Background The incidence rate of newly developed gallstone disease after gastrectomy for gastric cancer is thought to be higher than that in the general population. However, the presentation and management of these gallstones remain under debate, and the role of prophylactic cholecystectomy remains questionable.

Methods Data on adult patients who were diagnosed with gastric cancer and received gastrectomy between 2000 and 2011 were extracted from the Taiwan National Health Insurance Research Database. A patient was excluded if he or she had gallstone disease or received cholecystectomy before the index date. The incidence of newly developed gallstone disease and its subsequent management were recorded. Data were analyzed to evaluate the factors associated with gallstone development and treatment options.

Results A total of 17,325 gastric cancer patients who underwent gastrectomy were eligible for analysis. During the follow-up period (mean 4.1 years; median, 2.9 years), $1280(7.4 \%)$ patients developed gallstone disease and 560 (3.2\%) patients subsequently underwent cholecystectomy. The in-hospital mortality for cholecystectomy was $1.8 \%$ (10/560). Development of gallstone disease was associated

I-Shu Chen

nugaticc@gmail.com

1 Division of General Surgery, Department of Surgery, Kaohsiung Veterans General Hospital, No. 386, Dazhong 1st Rd, Zuoying District, Kaohsiung 81362, Taiwan

2 School of Medicine, National Yang-Ming University, Taipei, Taiwan

3 Critical Care Center and Cardiovascular Medical Center, Kaohsiung Veterans General Hospital, Kaohsiung, Taiwan
\end{abstract}

with older age, total gastrectomy, duodenal exclusion, diabetes, cirrhosis, and more comorbidities. Factors associated with the use of cholecystectomy to treat gallstone disease included younger age, fewer comorbidities, medical center admission, and presentation as cholecystitis.

Conclusions Although few patients required further gallbladder removal after gastrectomy for gastric malignancy, the increased mortality rate for subsequent cholecystectomy was worth noting. The decision to undergo prophylactic cholecystectomy might be individualized based upon patient characteristics and the surgeon's discretion.

Keywords Gallstone · Gastric cancer · Cholecystitis · Gastrectomy · Cholecystectomy

\section{Introduction}

Gastric cancer is the fifth most common malignant neoplasm worldwide, with half of the cases occurring in East Asia [1]. It also ranked as the third leading cause of cancerrelated death in 2012 [1]. Complete surgical resection of the gastric malignancy plus removal of adjacent lymph nodes is the best way to cure and achieve long-term survival [2]. However, several studies have shown a higher rate of gallstone formation after gastrectomy [3]. The reported incident rate was usually around $10-25 \%$, but rates as high as 47 and $60 \%$ had been cited in previous studies [3-5]. Possible mechanisms associated with this increased incidence included vagotomy, extent of gastrectomy, method of gastrointestinal reconstruction, and lymph node dissection [6, 7]. Some authors advocated prophylactic cholecystectomy, simultaneous removal of the gallbladder during gastric resection to prevent potential gallstone-related sequelae, such as cholecystitis, 
cholangitis, and pancreatitis [8]. This approach also avoided the possibility of subsequent cholecystectomy, which might be difficult owing to potential adhesion at the upper abdomen caused by previous gastrectomy and lymph node dissection [9]. A multicenter, randomized controlled trial, the Cholegas study, was initiated in November 2008 to evaluate prophylactic cholecystectomy; however, the final result has not been disclosed yet [10].

However, recent reports stated that the incidence rate of gallstone development after gastrectomy is not as high as we expected [11, 12]. Even if a gallstone develops after gastrectomy, it is usually asymptomatic, and its surgical removal is rarely needed [13]. Moreover, if cholecystectomy is indicated, it is still feasible to do it laparoscopically $[11,14]$. In this circumstance, it seems reasonable to preserve the gallbladder, and prophylactic cholecystectomy is not justified [3]. The debate remains unresolved because of the lack of a large-scale study; thus, no concrete suggestion can be provided.

The present study aimed to evaluate the incidence of newly developed gallstone disease after gastrectomy for gastric cancer, including its rate, associated factors, and management. Using a nationwide population-based data set, we analyzed sufficient patient data to answer this question.

\section{Methods}

\section{Patients and study design}

We performed a nationwide cohort study using administrative claims data obtained from the Taiwan National Health Insurance Research Database (NHIRD), a nationwide population data set that encompasses more than $99 \%$ of Taiwan residents. The accuracy of the NHIRD has been validated in many aspects of clinical studies [15-17]. All adult patients who were hospitalized with a primary diagnosis of gastric cancer [International Classification of Diseases, Ninth Edition, Clinical Modification (ICD-9CM) code 151] for the first time and had undergone gastrectomy without cholecystectomy between 1 January 2000 and 31 December 2011 comprised the study population (Table 1). The diagnostic accuracy of gastric cancer was confirmed by inclusion in the Registry for Catastrophic Illness Patients Database (RCIPD), a subset database that generally requires registration of the surgical pathology of patients. Patients could have copayment exemption once they were enrolled in RCIPD.

Patients diagnosed with gastric cancer before 1 January 2000 were excluded to ensure that the gastric cancer was newly diagnosed. Patients were also excluded if they were (1) younger than 18 years, (2) had a history of cholelithiasis, (3) had ever received cholecystectomy (either before
Table 1 Detail of ICD-9-CM diagnosis and procedure codes

\begin{tabular}{ll}
\hline Diagnosis/procedure & Codes \\
\hline Gastric cancer & $151 . x$ \\
Cholelithiasis & $574 . x, 575.0,575.1,575.6$ \\
With cholecystitis & $574.0,574.1,574.3,574.4,574.6$, \\
& $574.7,574.8,575.0,575.1$ \\
Without cholecystitis & $574.2,574.5,574.9,575.6$ \\
Gastrectomy & $43.5-43.9$ \\
Partial gastrectomy & $43.5-43.8$ \\
Total gastrectomy & 43.9 \\
Duodenal anastomosis & 43.6 \\
Duodenal exclusion & $43.5,43.7,43.8,43.9$ \\
Laparoscopy & 54.21 \\
Lymph node dissection & $40.29,40.3,40.50,40.52,40.59$ \\
Cholecystectomy & $51.22,51.23$ \\
Open & 51.22 \\
Laparoscopic & 51.23 \\
PTGBD & 51.01 \\
ERCP & 51.10 \\
\hline
\end{tabular}

$\overline{E R C P}$ endoscopic retrograde cholangiopancreatography; ICD-9-CM International Classification of Diseases, ninth edition clinical modification; PTGBD percutaneous transhepatic gallbladder drainage

the index date or concomitant), (4) had a history of hepatobiliary cancer, or (5) had died within 30 days of the index admission. Concomitant cholecystectomy is defined as the presence of a cholecystectomy code during the same hospitalization for gastrectomy.

This study was approved by the Human Research Committee of Kaohsiung Veterans General Hospital.

\section{Main outcome measurements}

Development of gallstone disease after gastrectomy was defined as rehospitalization with a diagnosis of cholelithiasis after the index hospitalization (Table 1). Managements of these newly developed gallstones, such as insertion of percutaneous transhepatic gallbladder drainage (PTGBD), endoscopic retrograde cholangiopancreatography (ERCP), or cholecystectomy, were also recorded. Nonoperative treatment was defined as no procedure coding of cholecystectomy during the rehospitalization period. The time interval to gallstone disease development and the interval to cholecystectomy were calculated from the date of the index hospitalization. All patients were followed up until death, withdrawal from the national health insurance program, or the end of 2013.

\section{Covariate assessment}

The type of gastrectomy was divided into partial and total gastrectomy. Post-gastrectomy reconstructions were 
categorized as duodenal anastomosis and duodenal exclusion. Laparoscopic gastrectomy was defined as the presence of a laparoscopy code in conjunction with coding for gastrectomy. Lymph node dissection is defined as the presence of relevant procedure codes and analyzed as a dichotomous variable (with or without lymph node dissection). Cholecystectomy was subcategorized as an open and laparoscopic approach. Because the surgical procedure code is associated with reimbursement that is paid by the national health insurance program, it is under strict scrutiny by medical reimbursement specialists, and a false insurance claim is punishable by significant fines, thus enhancing the accuracy of the coded information. Patients with cholelithiasis were further classified into groups with and without cholecystitis. Hospital levels were categorized as medical center, regional hospital, and district hospital.

\section{Statistical analysis}

To compare between different groups, the $\chi^{2}$ or Fisher's exact test was used for categorical variables, and the MannWhitney $U$ test was used for continuous variables. The cumulative incidence of gallstone formation after gastrectomy for gastric cancer was calculated using the KaplanMeier method. The factors that were possibly related to gallstone development were estimated with univariate and multivariate Cox proportional hazards regression model. A logistic regression model was used to analyze the associated factors for using cholecystectomy (versus nonoperative treatment) to treat newly developed gallstone disease. Hospital costs were obtained from the NHIRD. It included hospital room, laboratory, radiology, anesthetist, surgeon, operating room, pharmacy, special material, physical therapy, and miscellaneous costs during the designated hospitalization. Hospital costs were expressed in US dollars by conversion from New Taiwan dollars with the average exchange rate during the study period (1:30.5).

Statistical analyses were performed using IBM SPSS version 20 (IBM Corp., Armonk, NY, USA) and SAS 9.4 (SAS Institute, Inc., Cary, NC, USA). Statistical significance was defined as a $95 \%$ confidence interval (CI) not overlapping 1 and $p$ value $<0.05$.

\section{Results}

\section{Patient demographics}

After excluding patients with cholelithiasis or receiving cholecystectomy before the index date, a total of 17,325 gastric cancer patients who underwent gastrectomy for the first time between 2000 and 2011 were enrolled in this study (Fig. 1). Patient's median [interquartile range (IQR)] age was

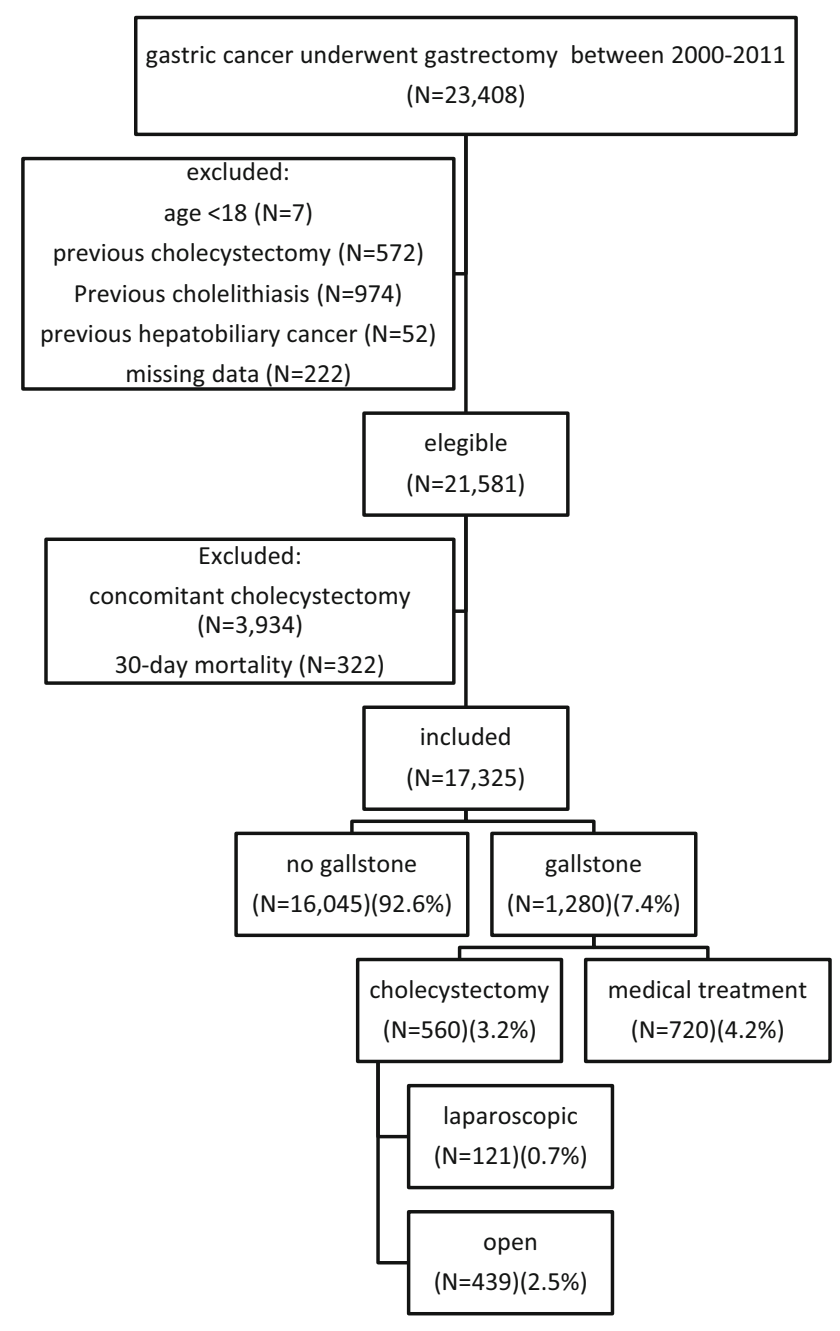

Fig. 1 Flowchart of subject selection

66.4 years (range 54.2-75.3 years), and $63.3 \%$ of the patients were male. Approximately half of the patients (49.5\%) were morbid, with a Charlson comorbidity index (CCI) $>2 ; 61.3 \%$ of the operations were performed in the medical center, and $68.9 \%$ of the patients underwent subtotal gastrectomy, whereas others received total gastrectomy. Duodenal anastomosis (Billroth I) accounted for only $8.4 \%$ of the patients. Lymph node dissection was done in half of the patients (51.2\%). The mean follow-up duration for this study was 4.1 years [standard deviation (SD), 3.7; median, 2.9; IQR 1.1-6.3 years]. During the study period, the overall death was $10,535(60.8 \%)$ patients. The 1-, 3-, and 5-year overall survival rates were $76.7,53.4$, and $44.3 \%$, respectively.

\section{Incidence of gallstone disease and its predictors}

During the follow-up period, 1280 (7.4\%) patients developed gallstone disease. The 1-, 2-, 5-, and 10-year cumulative incidence of gallstone formation was 2.2, 4.3, 9.5, and $15.7 \%$, respectively. Notably, the incidence curve does 


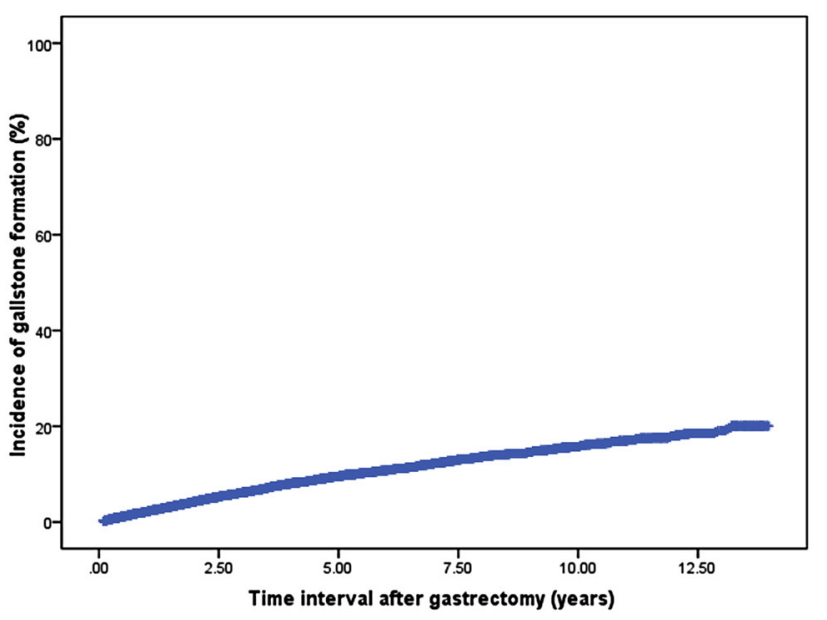

Fig. 2 Incidence of gallstone formation after gastrectomy

not end with a plateau yet (Fig. 2). The mean time to gallstone development was 3.1 years (SD, 2.8; median, 2.2; IQR $0.9-4.5$ years). Among those patients, 732 (57.2\%) presented with cholecystitis.

Table 2 shows a comparison between patients with and without cholelithiasis. Univariate and multivariate analyses showed that the development of gallstone disease was significantly associated with older age, total gastrectomy (versus subtotal gastrectomy), gastric anastomosis excluding duodenum, and presence of diabetes, cirrhosis, and more comorbidities (Table 3).

\section{Treatment options for gallstone disease}

Regarding gallstone management, PTGBD was inserted in $110(8.6 \%)$ patients. A total of $720(4.2 \%)$ patients were treated nonsurgically, and $560(3.2 \%)$ patients subsequently underwent cholecystectomy.

Cholecystectomy was done by an open and laparoscopic approach in 78.4 and $21.6 \%$ of the surgical patients, respectively. The mean time interval to cholecystectomy after gastric resection was 3.7 years (SD, 2.8; median, 3.0; IQR 1.5-5.2 years). The in-hospital mortality for cholecystectomy was ten patients (1.8\%).

Table 4 shows the characteristics and management of gallstone disease after gastrectomy. Age, CCI, and hospital level were significantly different between the cholecystectomy and nonoperative groups. Cholecystitis presented more in the operation group, whereas PTGBD was performed more in the nonoperative group. Both the cholecystectomy and nonoperative groups have similar percentages of patients who received ERCP (2.7 vs. $2.5 \%)$, respectively. Compared with the nonoperative group, the cholecystectomy group had a longer time interval for gallstone formation, longer hospital stay, and higher hospital cost.
On multivariate logistic regression models, medical center admission and presentation as cholecystitis were independently associated with an increased likelihood of receiving cholecystectomy rather than the nonoperative approach to treat those newly developed gallstone disease (Table 5). In contrast, older age and higher CCI were associated with a significantly lower preference for performing cholecystectomy for cholelithiasis. Sex, type of gastrectomy, laparoscopy use, lymph node dissection, DM, or cirrhosis did not have an influence on the treatment approach for newly developed gallstone disease.

\section{Discussion}

This study explored the incidence rate of newly developed gallstone disease and its management in patients with gastric cancer who underwent gastrectomy. By adopting nationwide population-based claim data, a sufficient number of patients with newly developed cholelithiasis $(N=1280)$ were retrieved for analysis. To our knowledge, this is currently the largest patient enrollment for this condition. We identified factors not only associated with gallstone development but also with the use of cholecystectomy to manage those gallstones.

The prevalence of gallstones in Taiwan's general population is around $4.3-14.4 \%$, and the prevalence of cholecystectomy for cholelithiasis is around 43.6-61.6 per 100,000 persons $[18,19]$. In our study, the incidence of newly developed gallstone disease is $7.4 \%$, which is comparable to two recent Korean studies [11, 12]. Based on their reports, gallstone disease occurred in 5.2\% (128/ $2480)$ and $7.2 \%$ (106/1480) of the gastric cancer patients who had undergone gastrectomy, respectively. However, note that the incidence curve of gallstone formation in our study did not end with a plateau yet; thus, the incidence of cholelithiasis may continue to increase if given more time, and additional years of follow-up is warranted (Fig. 2). Despite this, the incidence is lower compared with other earlier studies [3,5]. Racial disparity may be one of the reasons since cholelithiasis is known to be more prevalent in Western countries [20, 21]. In contrast, gallstone disease only affects $5-10 \%$ of the general population in East Asia $[22,23]$.

In a Japanese study done by Fukagawa et al. [13], 173 patients $(25.7 \%)$ developed gallstones, and $64.7 \%$ of the gallstones were detected within 1 year after gastrectomy postoperatively and an additional $19.1 \%$ of the gallstones were detected in the following year. Their data imply that more than $80 \%$ of the gallstones were formed within the first 2 years [4, 13]. Another study suggested that the lithogenesis caused by gastrectomy is transient [4]. Inoue et al. [24] reported that most of the gallbladder sludge 
Table 2 Characteristics of the enrolled patients

\begin{tabular}{|c|c|c|c|c|c|}
\hline \multirow[t]{2}{*}{ Characteristics } & \multicolumn{2}{|c|}{ Gallstone $(-), N=16,045$} & \multicolumn{2}{|c|}{ Gallstone $(+), N=1280$} & \multirow[t]{2}{*}{$p$} \\
\hline & $N$ & $(\%)$ & $N$ & $(\%)$ & \\
\hline $\operatorname{Age}^{\mathrm{a}}$ & 64.3 & $(13.7)$ & 66.5 & $(12.7)$ & $<0.001$ \\
\hline Sex & & & & & 0.672 \\
\hline Male & 10,156 & $(63.3)$ & 818 & $(63.9)$ & \\
\hline Female & 5889 & $(36.7)$ & 462 & $(36.1)$ & \\
\hline Type of gastrectomy & & & & & 0.007 \\
\hline Total gastrectomy & 4953 & $(30.9)$ & 442 & $(34.5)$ & \\
\hline Subtotal gastrectomy & 11,092 & $(69.1)$ & 838 & $(65.5)$ & \\
\hline Duodenal exclusion & & & & & $<0.001$ \\
\hline Yes & 14,666 & $(91.4)$ & 1207 & $(94.3)$ & \\
\hline No & 1379 & (8.6) & 73 & $(5.7)$ & \\
\hline Laparoscopy & & & & & 0.408 \\
\hline Yes & 634 & $(4.0)$ & 44 & (3.4) & \\
\hline No & 15,411 & $(96.0)$ & 1236 & $(96.6)$ & \\
\hline Lymph node dissection & & & & & 0.045 \\
\hline Yes & 8257 & $(51.5)$ & 621 & $(48.5)$ & \\
\hline No & 7788 & $(48.5)$ & 659 & $(51.5)$ & \\
\hline DM & & & & & 0.001 \\
\hline Yes & 2280 & $(14.2)$ & 225 & $(17.6)$ & \\
\hline No & 13,765 & $(85.8)$ & 1055 & $(82.4)$ & \\
\hline Cirrhosis & & & & & 0.036 \\
\hline Yes & 354 & $(2.2)$ & 40 & $(3.1)$ & \\
\hline No & 15,691 & $(97.8)$ & 1240 & $(96.9)$ & \\
\hline Charlson comorbidity index ${ }^{a}$ & 3.5 & $(3.2)$ & 3.1 & $(3.0)$ & 0.003 \\
\hline Hospital level & & & & & 0.815 \\
\hline Medical center & 9845 & $(61.4)$ & 781 & $(61.0)$ & \\
\hline Regional hospital & 5668 & $(35.3)$ & 460 & $(35.9)$ & \\
\hline Distric hospital & 532 & (3.3) & 39 & (3.0) & \\
\hline
\end{tabular}

$D M$ diabetes mellitus

${ }^{\text {a }}$ data presented as mean (standard deviation) detected in the early stage after gastrectomy will disappear within 1 year, along with the gradual recovery of gallbladder contractility. However, our study showed that the mean and median time intervals between gastrectomy and gallstone formation were 3.1 and 2.2 years, respectively, which were much longer than those of the Japanese study, and long-term surveillance might account for the late occurrence of gallstones.

In the present study, we noticed that gallstone formation was independently associated with older age, total gastrectomy, duodenal exclusion, diabetes, cirrhosis, and higher CCI. This is consistent with previous studies that reported gallstone formation was more likely in patients who received total rather than subtotal gastrectomy and those with gastrointestinal reconstruction that bypass the duodenum (i.e., Roux-en-Y, Billroth II) rather than anastomosing the residual stomach to the duodenum in end-toend fashion (Billroth I) [7].
Besides, many authors believe that lymph node dissection, especially the removal of lymph node in the hepatoduodenal ligament (station 12 lymph node), was associated with gallstone formation [7]. Damage of the nerve fiber that innervated the gallbladder in the hepatoduodenal ligament may be the underlying mechanism for this phenomenon [6]. However, the extent and detail of lymph node dissection done for the patients were not available in our database. This lack of information may be one of the reasons why our results show no significant association between lymph node dissection and gallstone formation.

Patients who develop gallstone disease after gastrectomy are usually treated medically or observed without surgery because the condition is usually asymptomatic $[7,13,25]$. This was also true in our study. Our study also showed that the different surgical treatments for gastric cancer (such as extent of gastric resection, method of gastrointestinal reconstruction, lymph node dissection or 
T. Liang et al.

Table 3 Factors associated with gallstone formation after gastrectomy

\begin{tabular}{|c|c|c|c|c|c|c|}
\hline \multirow[t]{2}{*}{ Variables } & \multicolumn{3}{|c|}{ Univariate } & \multicolumn{3}{|c|}{ Multivariate } \\
\hline & HR & $(95 \% \mathrm{CI})$ & $p$ & HR & $(95 \% \mathrm{CI})$ & $p$ \\
\hline Age & 1.025 & $(1.021-1.030)$ & $<0.001$ & 1.023 & $(1.018-1.028)$ & $<0.001$ \\
\hline Sex (male) & 1.131 & (1.009-1.268) & 0.035 & 1.019 & (0.908-1.143) & 0.747 \\
\hline \multicolumn{7}{|l|}{ Type of surgery } \\
\hline Total gastrectomy & 1.443 & $(1.286-1.620)$ & $<0.001$ & 1.297 & $(1.153-1.462)$ & $<0.001$ \\
\hline Duodenal exclusion & 2.168 & $(1.711-2.746)$ & $<0.001$ & 1.851 & $(1.457-2.355)$ & $<0.001$ \\
\hline Laparoscopy & 0.910 & $(0.673-1.230)$ & 0.539 & & & \\
\hline LN dissection & 0.846 & $(0.758-0.945)$ & 0.003 & 0.908 & $(0.812-1.015)$ & 0.091 \\
\hline DM & 1.482 & $(1.283-1.12)$ & $<0.001$ & 1.255 & $(1.082-1.455)$ & 0.003 \\
\hline Cirrhosis & 1.661 & $(1.212-2.277)$ & 0.002 & 1.572 & $(1.144-2.159)$ & 0.005 \\
\hline CCI & 1.051 & $(1.032-1.070)$ & $<0.001$ & 1.032 & $(1.013-1.051)$ & 0.001 \\
\hline \multicolumn{7}{|l|}{ Hospital level } \\
\hline Medical center & 0.860 & $(0.623-1.186)$ & 0.358 & & & \\
\hline Regional hospital & 0.962 & $(0.694-1.334)$ & 0.817 & & & \\
\hline District hospital & 1 & & & & & \\
\hline
\end{tabular}

not) do not appear to have any influence on the later management of gallstone disease. Multivariate analysis only found that younger age, fewer comorbidities, medical center admission, and presentation as cholecystitis were associated with the use of cholecystectomy instead of medical treatment for the management of newly developed gallstone disease.

Only $3.2 \%$ of our patients subsequently received cholecystectomy for their newly developed gallstone disease, and $21.6 \%(121 / 560)$ of the operations were done laparoscopically. Concerning the potential severe adhesion around the gallbladder, Lai et al. [25] reported that all of their subsequent cholecystectomies after gastrectomy were done directly through the open method. Nevertheless, Jun et al. [11] reported that in their series of late cholecystectomy after gastrectomy for gastric cancer, the gallbladder was removed laparoscopically in nearly half of their cases (49.2\%, 29/59). The laparoscopic group had shorter operation time, shorter hospital stay, and fewer complications compared with the open group, but patients in the open group were much older [11]. The proportion of common bile duct stones with/without cholangitis was also higher in the open group. Thus, it is unsurprising that during cholecystectomy, concurrent common bile duct exploration (choledochotomy) was done more frequently in the open group than in the laparoscopic group [11].

In our study, the in-hospital mortality of patients who received subsequent cholecystectomy for their newly developed gallstone disease was $1.8 \%$. The median age of these deceased patients was 75.3 years (IQR 70.8-83.6 years), which is 9 years older than the median age of the whole study population; $90 \%$ of the cases presented with cholecystitis, and all gallbladders were removed using the open approach. This high mortality rate for cholecystectomy was probably related to the patient's old age and to difficulty in tackling an inflamed gallbladder in an operative field that has been violated by previous gastrectomy and lymph node dissection.

However, the in-hospital mortality rate of patients who underwent nonsurgical management of gallstone disease was even higher $(6.8 \%, 49 / 720)$. Careful examination of the data showed that apart from the diagnosis of cholelithiasis, $27(55.1 \%)$ of the 49 deceased patients had metastatic lesions involving the liver, peritoneum, lymph node, bone, or lung. These findings may imply that many of the deaths were possibly more attributable to the progression of the underlying gastric cancer rather than to the management of gallstone disease.

Since the incidence of gallstone formation after gastrectomy was not very high in our study and most of the cases could be managed medically without cholecystectomy, the role of prophylactic cholecystectomy may not be justified. However, prophylactic cholecystectomy may be beneficial in a non-Asian population where the incidence of gallstones is much higher. It may also be considered in patients who have undergone Roux-en-Y reconstruction because ERCP would be difficult to conduct if a common bile duct stone develops.

In the Cholegas study, adding cholecystectomy to gastric cancer surgery did not significantly affect perioperative morbidity, mortality, and costs. However, one case $(1.5 \%)$ of biliary leakage that was probably caused by prophylactic 
Table 4 management of gallstone disease after gastrectomy

\begin{tabular}{|c|c|c|c|c|c|}
\hline \multirow[t]{2}{*}{ Characteristics } & \multicolumn{2}{|c|}{ Cholecystectomy $(N=560)$} & \multicolumn{2}{|c|}{ No operation $(N=720)$} & \multirow[t]{2}{*}{$p$} \\
\hline & $N$ & $\%$ & $N$ & $\%$ & \\
\hline $\operatorname{Age}^{a}$ & 65.1 & $(12.0)$ & 67.6 & $(13.1)$ & $<0.001$ \\
\hline Sex & & & & & 0.639 \\
\hline Male & 362 & $(64.6)$ & 456 & $(63.3)$ & \\
\hline Female & 198 & $(35.4)$ & 264 & $(36.7)$ & \\
\hline Type of gastrectomy & & & & & 0.343 \\
\hline Total gastrectomy & 185 & $(33.0)$ & 257 & $(35.7)$ & \\
\hline Subtotal gastrectomy & 375 & $(67.0)$ & 463 & $(64.3)$ & \\
\hline Duodenal exclusion & & & & & 0.903 \\
\hline Yes & 529 & (94.5) & 678 & $(94.2)$ & \\
\hline No & 31 & $(5.5)$ & 42 & $(5.8)$ & \\
\hline Laparoscopy & & & & & 0.164 \\
\hline Yes & 24 & (4.3) & 20 & $(2.8)$ & \\
\hline No & 536 & $(95.7)$ & 700 & $(97.2)$ & \\
\hline Lymph node dissection & & & & & 0.176 \\
\hline No & 276 & $(49.3)$ & 383 & $(53.2)$ & \\
\hline Yes & 284 & $(50.7)$ & 337 & $(46.8)$ & \\
\hline $\mathrm{DM}$ & & & & & 0.300 \\
\hline Yes & 91 & $(16.2)$ & 134 & $(18.6)$ & \\
\hline No & 469 & $(83.8)$ & 586 & $(81.4)$ & \\
\hline Cirrhosis & & & & & 0.747 \\
\hline Yes & 16 & $(2.9)$ & 24 & (3.3) & \\
\hline No & 544 & $(97.1)$ & 696 & $(96.7)$ & \\
\hline Charlson comorbidity index ${ }^{a}$ & 2.8 & $(2.9)$ & 3.3 & $(3.0)$ & 0.003 \\
\hline Hospital level & & & & & 0.026 \\
\hline Medical center & 358 & $(63.9)$ & 423 & $(58.8)$ & \\
\hline Regional hospital & 192 & $(34.3)$ & 268 & $(37.2)$ & \\
\hline Distric hospital & 10 & $(1.8)$ & 29 & $(4.0)$ & \\
\hline Cholecystitis & & & & & $<0.001$ \\
\hline Yes & 398 & $(71.1)$ & 334 & $(46.4)$ & \\
\hline No & 162 & $(28.9)$ & 386 & $(53.6)$ & \\
\hline PTGBD & & & & & 0.009 \\
\hline Yes & 35 & $(6.2)$ & 75 & $(10.4)$ & \\
\hline No & 525 & $(93.8)$ & 645 & $(89.6)$ & \\
\hline ERCP & & & & & 0.860 \\
\hline Yes & 14 & $(2.7)$ & 18 & $(2.5)$ & \\
\hline No & 545 & $(97.3)$ & 702 & $(97.5)$ & \\
\hline Time to gallstone formation ${ }^{\mathrm{a}}$, year & 3.5 & $(2.8)$ & 2.8 & $(2.7)$ & $<0.001$ \\
\hline 2nd Length of stay ${ }^{\mathrm{a}}$, day & 15.3 & $(21.5)$ & 11.4 & $(16.4)$ & $<0.001$ \\
\hline 2nd Hospital $\operatorname{cost}^{\mathrm{a}}$, USD & 4006.9 & $(5783.3)$ & 2027.3 & $(4036.2)$ & $<0.001$ \\
\hline In-hospital mortality & 10 & $(1.8)$ & 49 & $(6.8)$ & $<0.001$ \\
\hline
\end{tabular}

$d$ day, DM diabetes mellitus, ERCP endoscopic retrograde cholangiopancreatography, PTGBD percutaneous transhepatic gallbladder drainage, USD United States dollars, $y$ year

${ }^{a}$ Data presented as mean (standard deviation) cholecystectomy was observed [10]. Another Japanese study concluded that the addition of laparoscopic cholecystectomy did not alter complications, mortality, and length of stay in patients undergoing laparoscopic gastrectomy for gastric cancer. Nevertheless, the medical cost was significantly higher [26].

In Taiwan, the average length of hospital stay for open and laparoscopic cholecystectomy is 13.1 and 4.9 days, 
Table 5 Factor associated with cholecystectomy for gallstone (versus no oepration)

\begin{tabular}{|c|c|c|c|c|c|c|}
\hline \multirow[t]{2}{*}{ Variables } & \multicolumn{3}{|c|}{ Univariate } & \multicolumn{3}{|c|}{ Multivariate } \\
\hline & OR & $(95 \% \mathrm{CI})$ & $p$ & OR & $(95 \% \mathrm{CI})$ & $p$ \\
\hline Age & 0.985 & $(0.976-0.993)$ & 0.001 & 0.984 & $(0.975-0.993)$ & $<0.001$ \\
\hline Sex (male) & 0.945 & $(0.750-1.189)$ & 0.628 & & & \\
\hline \multicolumn{7}{|l|}{ Type of surgery } \\
\hline Total gastrectomy & 0.889 & $(0.704-1.122)$ & 0.321 & & & \\
\hline Duodenal exclusion & 1.057 & $(0.656-1.705)$ & 0.820 & & & \\
\hline Laparoscopy & 1.567 & $(0.857-2.867)$ & 0.145 & & & \\
\hline LN dissection & 1.169 & $(0.937-1.459)$ & 0.165 & & & \\
\hline $\mathrm{DM}$ & 0.849 & $(0.633-1.137)$ & 0.271 & & & \\
\hline Cirrhosis & 0.853 & $(0.449-1.622)$ & 0.627 & & & \\
\hline $\mathrm{CCI}$ & 0.944 & (0.909-0.980) & 0.003 & 0.930 & $(0.894-0.968)$ & $<0.001$ \\
\hline \multicolumn{7}{|l|}{ Hospital level } \\
\hline Medical center & 2.454 & $(1.180-5.105)$ & 0.016 & 2.534 & $(1.179-5.446)$ & 0.017 \\
\hline Regional hospital & 2.078 & $(0.989-4.364)$ & 0.054 & 2.059 & $(0.950-4.463)$ & 0.067 \\
\hline Distric hospital & 1 & & & & & \\
\hline Cholecystitis & 2.839 & $(2.247-3.588)$ & $<0.001$ & 2.988 & $(2.353-3.795)$ & $<0.001$ \\
\hline
\end{tabular}

$C C I$ Charlson comorbidity index, $C I$ confidence interval, $D M$ diabetes mellitus, $L N$ lymph node, $O R$ odds ratio respectively, which is longer than that reported in other countries, not to mention those performing laparoscopic cholecystectomy as an outpatient procedure $[19,27]$. The longer hospital stay in Taiwan may be related to the different treatment protocol or cultural difference [19]. In our study, the mean hospital stay for cholecystectomy is 15.3 days (open, 17.5; laparoscopic, 7.3). Despite the longer stay, the copayment, which is usually $10 \%$ of the total cost, is affordable for most patients in Taiwan.

This study has several limitations. First, the definition of gallstone formation was based on the diagnostic coding of the inpatient database. If gallstone formation was detected through an imaging study arranged in the outpatient clinic and by chance the patient did not indicate any subsequent management (i.e., gastric cancer-related adjuvant therapy, follow-up, or treatment of cholelithiasis), we might not be able to identify those newly developed gallstones, thus underestimating the incidence of cholelithiasis. Nevertheless, the lack of hospitalization might also imply that the gallstone is asymptomatic or clinically insignificant. Therefore, this limitation did not compromise our main results. Second, details of lymph node dissection were not available in our database, such as the extent of lymph node dissection (i.e., D0, D1, and D2) and dissection in the hepatoduodenal ligament (station 12 lymph node). Thus, we were unable to analyze further the impact of lymph node dissection on the development of cholelithiasis. Third, the tumor stage of gastric cancer was not recorded in our database. However, about half of the patients underwent gastrectomy without lymph node dissection in our study, and $60 \%$ of our patients died during the study period. These findings might imply that a certain proportion of the enrolled patients were in the advanced stage and received palliative surgery. Fourth, our study intended to exclude patients who received concomitant cholecystectomy in the first place. However, the coding accuracy is likely lower since cholecystectomy is a secondary procedure performed in addition to the gastrectomy and inclusion of patients who already had their gallbladders removed would underestimate the real incidence of cholelithiasis.

In conclusion, the incidence of cholelithiasis after gastrectomy for gastric cancer was not very high, and less than half of those cases require further surgery to remove the gallbladder. However, the increased mortality rate for the subsequent cholecystectomy was worth noting. Therefore, the decision to undergo prophylactic cholecystectomy might be individualized based upon patient characteristics and the surgeon's discretion.

Acknowledgements The authors express their appreciation to the Research Center of Medical Informatics in Kaohsiung Veterans General Hospital for assistance in data processing.

\section{Compliance with ethical standards}

Conflict of interest The authors declare that they have no conflict of interest.

Human rights statement and informed consent All procedures followed were in accordance with the ethical standards of the responsible committee on human experimentation (institutional and national) and with the Helsinki Declaration of 1964 and later versions. The Taiwan National Health Insurance Research Database contained 
unidentifiable secondary data. Information that might identify the individual patients was all encrypted. This study was approved by the Human Research Committee of Kaohsiung Veterans General Hospital.

\section{References}

1. Ferlay J, Soerjomataram I, Dikshit R, Eser S, Mathers C, Rebelo $\mathrm{M}$, et al. Cancer incidence and mortality worldwide: sources, methods and major patterns in GLOBOCAN 2012. Int J Cancer. 2015;136(5):E359-86.

2. Allum WH, Blazeby JM, Griffin SM, Cunningham D, Jankowski JA, Wong R, et al. Guidelines for the management of oesophageal and gastric cancer. Gut. 2011;60(11):1449-72.

3. Gillen S, Michalski CW, Schuster T, Feith M, Friess H, Kleeff J. Simultaneous/Incidental cholecystectomy during gastric/esophageal resection: systematic analysis of risks and benefits. World J Surg. 2010;34(5):1008-14.

4. Hauters P, de Neve de Roden A, Pourbaix A, Aupaix F, Coumans P, Therasse G. Cholelithiasis: a serious complication after total gastrectomy. Br J Surg. 1988;75(9):899-900.

5. Bianchi A, Sunol J, Casals S, Badia A, Ubach M. Cholelithiasis after total gastrectomy for gastric cancer. Rev Esp Enferm Dig. 1994;85(2):91-3.

6. Yi SQ, Ohta T, Tsuchida A, Terayama H, Naito M, Li J, et al. Surgical anatomy of innervation of the gallbladder in humans and Suncus murinus with special reference to morphological understanding of gallstone formation after gastrectomy. World J Gastroenterol. 2007;13(14):2066-71.

7. Kobayashi T, Hisanaga M, Kanehiro H, Yamada Y, Ko S, Nakajima Y. Analysis of risk factors for the development of gallstones after gastrectomy. Br J Surg. 2005;92(11):1399-403.

8. Wu CC, Chen CY, Wu TC, Iiu TJ, P'Eng PK. Cholelithiasis and cholecystitis after gastrectomy for gastric carcinoma: a comparison of lymphadenectomy of varying extent. Hepatogastroenterology. 1995;42(6):867-72.

9. Sasaki A, Nakajima J, Nitta H, Obuchi T, Baba S, Wakabayashi G. Laparoscopic cholecystectomy in patients with a history of gastrectomy. Surg Today. 2008;38(9):790-4.

10. Bernini M, Bencini L, Sacchetti R, Marchet A, Cristadoro L, Pacelli F, et al. The Cholegas study: safety of prophylactic cholecystectomy during gastrectomy for cancer: preliminary results of a multicentric randomized clinical trial. Gastric Cancer. 2013;16(3):370-6.

11. Jun KH, Kim JH, Kim JJ, Chin HM, Park SM. Retrospective analysis on the gallstone disease after gastrectomy for gastric cancer. Gastroenterol Res Pract. 2015;2015:827864.

12. Paik KH, Lee JC, Kim HW, Kang J, Lee YS, Hwang JH, et al. Risk factors for gallstone formation in resected gastric cancer patients. Medicine (Baltimore). 2016;95(15):e3157.

13. Fukagawa T, Katai H, Saka M, Morita S, Sano T, Sasako M. Gallstone formation after gastric cancer surgery. J Gastrointest Surg. 2009;13(5):886-9.
14. Kwon AH, Inui $\mathrm{H}$, Imamura A, Kaibori M, Kamiyama $\mathrm{Y}$. Laparoscopic cholecystectomy and choledocholithotomy in patients with a previous gastrectomy. J Am Coll Surg. 2001;193(6):614-9.

15. Cheng CL, Kao YH, Lin SJ, Lee CH, Lai ML. Validation of the national health insurance research database with ischemic stroke cases in Taiwan. Pharmacoepidemiol Drug Saf. 2011;20(3):236-42.

16. Cheng CL, Lee CH, Chen PS, Li YH, Lin SJ, Yang YH. Validation of acute myocardial infarction cases in the national health insurance research database in taiwan. $\mathrm{J}$ Epidemiol. 2014;24(6):500-7.

17. Cheng CL, Chien HC, Lee CH, Lin SJ, Yang YK. Validity of inhospital mortality data among patients with acute myocardial infarction or stroke in national health insurance research database in Taiwan. Int J Cardiol. 2015;201:96-101.

18. Hung SC, Liao KF, Lai SW, Li CI, Chen WC. Risk factors associated with symptomatic cholelithiasis in Taiwan: a population-based study. BMC Gastroenterol. 2011;11:111.

19. Hsu CE, Lee KT, Chang CS, Chiu HC, Chao FT, Shi HY. Cholecystectomy prevalence and treatment cost: an 8-year study in Taiwan. Surg Endosc. 2010;24(12):3127-33.

20. Shaffer EA. Gallstone disease: epidemiology of gallbladder stone disease. Best Pract Res Clin Gastroenterol. 2006;20(6):981-96.

21. Everhart JE, Yeh F, Lee ET, Hill MC, Fabsitz R, Howard BV, et al. Prevalence of gallbladder disease in American Indian populations: findings from the strong heart study. Hepatology. 2002;35(6):1507-12.

22. Huang J, Chang CH, Wang JL, Kuo HK, Lin JW, Shau WY, et al. Nationwide epidemiological study of severe gallstone disease in Taiwan. BMC Gastroenterol. 2009;9:63.

23. Nomura H, Kashiwagi S, Hayashi J, Kajiyama W, Ikematsu H, Noguchi A, et al. Prevalence of gallstone disease in a general population of Okinawa, Japan. Am J Epidemiol. 1988;128(3):598-605.

24. Inoue K, Fuchigami A, Higashide S, Sumi S, Kogire M, Suzuki $\mathrm{T}$, et al. Gallbladder sludge and stone formation in relation to contractile function after gastrectomy. A prospective study. Ann Surg. 1992;215(1):19-26.

25. Lai SL, Yang JC, Wu JM, Lai IR, Chen CN, Lin MT, et al. Combined cholecystectomy in gastric cancer surgery. Int J Surg. 2013;11(4):305-8.

26. Murata A, Okamoto K, Muramatsu K, Kubo T, Fujino Y, Matsuda S. Effects of additional laparoscopic cholecystectomy on outcomes of laparoscopic gastrectomy in patients with gastric cancer based on a national administrative database. J Surg Res. 2014;186(1):157-63.

27. Ahmad NZ, Byrnes G, Naqvi SA. A meta-analysis of ambulatory versus inpatient laparoscopic cholecystectomy. Surg Endosc. 2008;22(9):1928-34. 